\title{
All-Optical Polarization Control for Telecom Applications
}

\author{
P-Y. Bony ${ }^{1}$, M. Guasoni ${ }^{1}$, S. Pitois ${ }^{1}$, A. Picozzi ${ }^{1}$, D. Sugny ${ }^{1}$, H. Jauslin ${ }^{1}$, G. Millot ${ }^{1}$, S. Wabnitz ${ }^{2}$ and J. Fatome ${ }^{1}$ \\ 1. Laboratoire Interdisciplinaire Carnot de Bourgogne, UMR 6303 CNRS-Université de Bourgogne, 9 Av. A. Savary, 21078 Dijon, France \\ 2. Department of Information Engineering, Università di Brescia, Via Branze 38, 25123 Brescia, Italy \\ ifatome@u-bourgogne.fr
}

\begin{abstract}
We describe a phenomenon of self-organization of the light state-of-polarization in optical fibers based on a nonlinear cross-polarization interaction between an incident signal and its backward replica. Several proof-of-principles for telecom applications are reported.

OCIS codes: (060.0060) Fiber optics and optical communications; (060.4370) Nonlinear optics, fibers; (190.4380) Nonlinear optics, four-wave mixing; (230.4320) Nonlinear optical devices; (250.4745) Optical processing devices
\end{abstract}

\section{Introduction}

In many fields of photonics, especially in fiber-based systems, the light state-of-polarization (SOP) remains one of the most elusive and underexploited parameter that is still challenging to fix and to control [1,2]. Indeed, despite the impressive progress of manufacturing process of standard optical fibers, the residual birefringence combined with environmental variations and local mechanical stress such as bending, squeezing, vibrations or temperature fluctuations make the SOP of a light beam completely random and unpredictable after a few hundreds of meters of propagation. In this context, and despite the high efficiency of digital signal processing and sophisticated algorithms implemented in current coherent transmission systems, all-optical control of light SOP still remains an open issue, which could lead to new functionalities for future optical systems.

To this aim, controlling the SOP of an incident signal thanks to an external pump wave has been a subject of high interest and intense research [3-18]. In order to surpass standard polarizers which vanish up to $50 \%$ of an incoming unpolarized light and intrinsically transfer SOP variations into intensity fluctuations, several ideas have emerged to develop an "ideal polarizer" which could lead to a complete control of the light SOP with $100 \%$ efficiency. This phenomenon of polarization pulling or trapping has been the subject of several works, exploiting the polarization dependence of the Raman gain [3-6] and Brillouin scattering [7-9], the parametric amplification [10] as well as a counter-propagating four-wave mixing process [11-14].

The common drawback of these techniques is that a fully polarized pump beam is used as polarization reference for the incoming signal and therefore has to be accurately stabilized in turn. To overcome this issue, we explore a different approach and exploit the unique capability for a light beam to self-organize its own SOP, upon nonlinear propagation in optical fibers, into universal and environmentally robust states [15]. This nonlinear device, called Omnipolarizer, is able to self-organize its SOP without any need of additional control and provides a wide range of dynamics, which allowed us to experimentally demonstrate several optical functionalities for Telecom applications such as polarization funnel and digital polarization-beam-splitter [15], optical flip-flop memory and all-optical polarization switch [16] as well as polarization insensitive parametric amplifier [17] and all-optical scrambler [18].

\section{Principle}

The principle of operation of the Omnipolarizer is depicted in Fig. 1. Basically, it consists in a single span of optical fiber, where a signal beam nonlinearly interacts through a four-wave-mixing process with its own counterpropagating replica generated at fiber end by means of an amplified reflective loop setup.

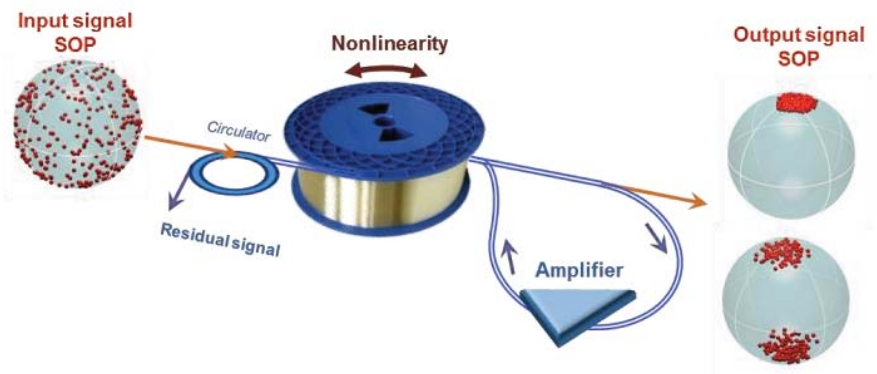

Fig. 1. Principle of the Omnipolarizer

The dynamics of that system is mainly driven by the reflective coefficient of the loop $g$ defined by the power ratio between the backward and forward signals at fiber end. In practice, this coefficient $g$ is directly controlled by 
the power ratio between the output and input Erbium-doped fiber amplifiers (EDFA). Depending on that coefficient, the Omnipolarizer may operate in, and be switched between three distinct operating modes: a digital polarization beam splitter (PBS), a nonlinear ideal polarizer or a fully chaotic polarization scrambler.

More precisely, the dynamics of the Stokes vectors in the Omnipolarizer are related to the stability of the stationary states of the system, which are ruled by the solutions of a set of two coupled nonlinear Schrödinger equations in the continuous wave limit, i.e. when dropping the time derivatives [19]. Basically, only the stationary solutions which exhibit a non-oscillatory evolution along the fiber length are stable and can play the role of natural attractors for the output SOP [19-21]. This fundamental rule allows us to point out the values of $g$ for which the Omnipolarizer switches between the three operating regimes and leads to define the following threshold $g_{A}=9 \pi /[16 L P(L) \gamma]$, where $L$ is the fiber length, $P(L)$ the incident signal power at fiber end and $\gamma$ the nonlinear Kerr coefficient. For $g$ just below unity, the device is characterized by a bistability associated to a hysteresis cycle and digitalizes the output SOP along two orthogonal states. In that case, the initial ellipticity determines which of the two pools of attraction is obtained. For $1<g<g_{A}$, only one point of attraction survives and the Omnipolarizer behaves as an ideal polarizer. If $g>10 g_{A}$, then the system becomes fully chaotic and plays the role of an all-optical scrambler. Furthermore, for $g_{A}<g<10 g_{A}$, a transient regime is then experienced for which fixed points, periodic trajectories or even partially chaotic regimes can be observed without any possible prediction and control.

\section{Experimental results}

The experimental setup and results for the two first operating modes are summarized in Fig. 2. A polarizationscrambled 40-Gbit/s On/Off Keying signal is first generated at $1564 \mathrm{~nm}$. This arbitrary polarized signal is then amplified by means of the input EDFA until $27 \mathrm{dBm}$ before injection into a 6.2-km long non-zero dispersion fiber span (NZ-DSF) characterized by a chromatic dispersion $D=-1.5 \mathrm{ps} / \mathrm{nm} / \mathrm{km}$ at $1550 \mathrm{~nm}$ and a nonlinear coefficient $\gamma=1.7 \mathrm{~W}^{-1} \mathrm{~km}^{-1}$. The resulting incident signal then nonlinearly interacts with its own counter-propagating replica generated at the fiber output by means of a reflective loop setup made of an optical circulator and a second EDFA. A 90/10 coupler is also added into the back-reflective loop to collect the stabilized signal.

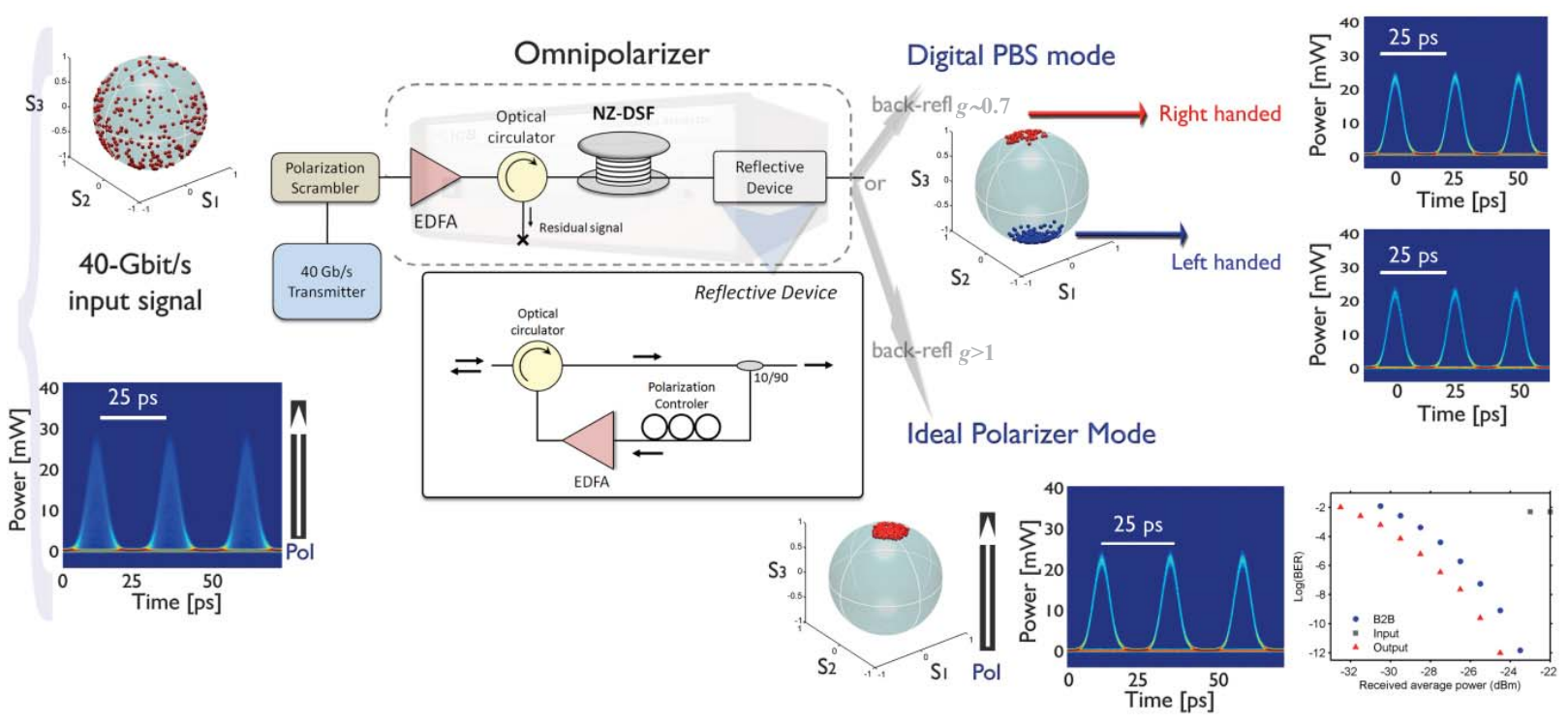

Fig. 2. Experimental setup and results of the Omnipolarizer for two working regimes: ideal polarizer mode and digital PBS mode.

Depending on the back-reflective coefficient $g$, adjustable thanks to the gain of the second EDFA, the two first working regimes can be achieved. As illustrated in Fig. 2, when $g$ is slightly larger than 1 (i.e., the backward signal is amplified up to $28 \mathrm{dBm}$ ), any arbitrary input SOP converges on the Poincaré sphere towards a fixed and unique output SOP. The Omnipolarizer acts as a powerful polarization funnel which enables to reach a very high degree-ofpolarization close to 1 . This behavior is even more striking in the temporal domain. Indeed, at the input of the device, because of the polarization scrambling process, after detection of the signal beyond a polarizer, all the initial polarization variations are transferred into large intensity fluctuations leading to a strongly closed eye-diagram of the 40-Gbit/s signal. On the contrary, at the output of the Omnipolarizer, because of the self-repolarization effect, and despite the initial scrambling process, the resulting eye-diagram remains completely opened beyond the polarizer, indicating that the polarization attraction process operates in full strength. Furthermore, despite the polarization 
scrambling and the drop of the BER to a threshold around $10^{-2}$ at the input of the system when detected beyond a polarizer (grey squares), the self-repolarization effect (red triangles) allows us to completely recover the initial data (back-to-back in blue circles).

The PBS operating mode can be reached when the reflective coefficient is now decreased just below unity, here $g \sim 0.7$ (backward power of $25 \mathrm{dBm}$ ). In that case, any arbitrary input SOP is digitally attracted towards two orthogonal SOPs depending exclusively on the value of the initial ellipticity. Two small attraction pools are clearly visible on the output Poincaré sphere corresponding here to the north (right handed SOP) and south poles (lefthanded). The device becomes indeed bistable and its output polarization is characterized by a phenomenon of hysteresis [16]. As a result, we obtain a discrete polarization beam splitter which enable to efficiently route $40-\mathrm{Gbit} / \mathrm{s}$ data packets on one or the other orthogonal axis with an excellent extinction ratio (larger than $20 \mathrm{~dB}$ ).

\section{Conclusion}

In this work, we report the experimental observation of the self-organization of the light state-of-polarization in optical fibers. The present device called Omnipolarizer is based on the nonlinear cross-polarization interaction in a standard optical fiber between a forward signal and its counter-propagating replica, generated at fiber end by means of a reflective loop setup. The Omnipolarizer offers a wide range of dynamics which allowed us to highlight three distinct operating regimes. The digital PBS mode, characterized by a SOP bistability, has been exploited to develop a 40-Gbit/s digital polarization-beam-splitter and an optical flip-flop memory $[15,16]$. The ideal polarizer mode for which the device behaves as a polarization funnel and enables to trap any output SOP. Finally, the chaotic mode for which the Omnipolarizer acts as a fast and fully nondeterministic all-optical polarization scrambler. This work is funded by the ERC under the FP7 Grant Agreement n 306633, PETAL project [22].

\section{References}

[1] M. Martinelli, P. Martelli, and S. M. Pietralunga, "Polarization stabilization in optical communications systems," J. Lightw. Technol. 24, 4172-4183 (2006).

[2] M. Boroditsky, M. Brodsky, N. J. Frigo, P. Magill, and H. Rosenfeldt, "Polarization dynamics in installed fiberoptic systems," in IEEE LEOS Annual Meeting Conference Proceedings (LEOS), 413-414 (2005).

[3] M. Martinelli, M. Cirigliano, M. Ferrario, L. Marazzi, and P. Martelli, "Evidence of Raman-induced polarization pulling,” Opt. Express 17, 947-955 (2009).

[4] L. Ursini, M. Santagiustina, and L. Palmieri, "Raman Nonlinear Polarization Pulling in the Pump Depleted Regime in Randomly Birefringent Fibers," IEEE Photon. Technol. Lett. 23, 1041-1135 (2011).

[5] S. Sergeyev and S. Popov, "Two-section fiber optic Raman polarizer," IEEE J. Quantum Electron. 48, 56-60 (2012).

[6] N. J. Muga, M. F. S. Ferreira, and A. N. Pinto, "Broadband polarization pulling using Raman amplification," Opt. Express 19, 18707-18712 (2011).

[7] L. Thevenaz, A. Zadok, A. Eyal and M. Tur, "All-optical polarization control through Brillouin amplification", in Optical Fiber Communication Conference, OFC'08, paper OML7 (2008).

[8] A. Zadok, E. Zilka, A. Eyal, L. Thévenaz, and M. Tur, "Vector analysis of stimulated Brillouin scattering amplification in standard singlemode fibers," Opt. Express 16, 21692-21707 (2008).

[9] J. Fatome, S. Pitois, and G. Millot, "Experimental evidence of Brillouin-induced polarization wheeling in highly birefringent optical fibers," Opt. Express 17, 12612-12618 (2009).

[10] B. Stiller, P. Morin, D. M. Nguyen, J. Fatome, S. Pitois, E. Lantz, H. Maillotte, C. R. Menyuk, and T. Sylvestre, "Demonstration of polarization pulling using a fiber-optic parametric amplifier," Opt. Express 20, 27248-27253 (2012).

[11] J. Fatome, S. Pitois, P. Morin, and G. Millot, "Observation of light-by-light polarization control and stabilization in optical fibre for telecommunication applications," Opt. Express 18, 15311-15317 (2010).

[12] P. Morin, J. Fatome, C. Finot, S. Pitois, R. Claveau, and G. Millot, "All-optical nonlinear processing of both polarization state and intensity profile for $40 \mathrm{Gbit} / \mathrm{s}$ regeneration applications," Opt. Express 19, 17158-17166 (2011).

[13] V. V. Kozlov, J. Fatome, P. Morin, S. Pitois, G. Millot, and S. Wabnitz, "Nonlinear repolarization dynamics in optical fibers: transient polarization attraction,” J. Opt. Soc. Am. B 28, 1782-1791 (2011).

[14] Matteo Barozzi, and Armando Vannucci, "Lossless Polarization Attraction of Telecom Signals: Application to All-Optical OSNR Enhancement," J. Opt. Soc. Am. B, in press, Doc. ID 217763, (2014).

[15] J. Fatome, S. Pitois, P. Morin, E. Assemat, D. Sugny, A. Picozzi, H. R. Jauslin, G. Millot, V. V. Kozlov, and S. Wabnitz, "A universal optical all-fiber omnipolarizer," Sci. Rep., 2, 938; DOI:10.1038/srep00938 (2012).

[16] P.-Y. Bony, M. Guasoni, E. Assémat, S. Pitois, D. Sugny, A. Picozzi, H. R. Jauslin, and J. Fatome, Optical flip-flop memory and data packet switching operation based on polarization bistability in a telecommunication optical fiber, J. Opt. Soc. Am. B, 30, pp. 2318-2325, (2013).

[17] P. Y. Bony, M. Guasoni, P. Morin, D. Sugny, A. Picozzi, H. R. Jauslin, S. Pitois and J. Fatome,"Temporal spying and concealing process in fibre-optic data transmission systems through polarization bypass", Nat. Commun. 5:4678 doi: 10.1038/ncomms5678 (2014).

[18] M. Guasoni, P.-Y. Bony, S. Pitois, D. Sugny, A. Picozzi, H.-R. Jauslin, and J. Fatome, "Fast Polarization Scrambler Based on Chaotic Dynamics in Optical Fibers," in European Conference on Optical Communications ECOC 2014, Cannes, paper Tu.1.4.5 (2014).

[19] V.V. Kozlov, J. Nuño, and S. Wabnitz, "Theory of lossless polarization attraction in telecommunication fibers," J. Opt. Soc. Am. B 28, 100$108(2011)$.

[20] E. Assémat, A. Picozzi, H. R. Jauslin, and D.Sugny, "Hamiltonian tools for the analysis of optical polarization control,” J. Opt. Soc. Am. B 29, 559-571 (2012).

[21] K. S. Turitsyn and S. Wabnitz, "Stability analysis of polarization attraction in optical fibers," Opt. Commun. 307, $62-66$ (2013).

[22] https://www.facebook.com/petal.inside 